\title{
Transesophageal Echocardiogram as a Diagnostic Tool for Cardioembolic Cerebrovascular Event Secondary to Thrombus into Valsalva Sinus Aneurysm
}

\author{
Cesar Fernando Somoza ${ }^{1^{*}}$, Jose Fernando Zuluaga ${ }^{2}$ and Jaime Rodriguez ${ }^{3}$ \\ ${ }^{1}$ Echocardiography fellowship, Universidad El Rosario, School of Medicine and Health Sciences, Colombia \\ ${ }^{2}$ Department of Cardiology, Fundacion Clinica Shaio, Shaio, Colombia
}

${ }^{3}$ Department of Cardiology, Universidad El Rosario, Fundacion Clinica Shaio, Colombia

"Corresponding author: Cesar Fernando Somoza, Universidad El Rosario, School of Medicine and Health Sciences, Colombia, Tel: 50496831877; E-mail: cfsomoza@outlook.com

Received: June 12, 2018; Accepted: July 11, 2018; Published: July 14, 2018

Copyright: ( 2018 Somoza CF, et al. This is an open-access article distributed under the terms of the creative commons attribution license, which permits unrestricted use, distribution, and reproduction in any medium, provided the original author and source are credited.

\begin{abstract}
Transesophageal echocardiogram as a diagnostic tool for cardioembolic cerebrovascular event secondary to thrombus into Valsalva sinus aneurysm. This is a case report of a 54-years-old female with a history of systemic arterial hypertension treated with enalapril $20 \mathrm{mg}$ and amlodipine $5 \mathrm{mg}$ both once daily. She was admitted 15 days ago in a Hospital Center of Bogotá for having presented sudden loss of consciousness followed by complex focal seizure episodes. She presented with motor and sensory aphasia and left hemiparesis, atrial fibrillation was documented in electrocardiogram and an ischemic cardiovascular event was documented in a brain computer tomography. A cardioembolic event due atrial fibrillation was considered and for that reason the patient was referred to the Shaio Clinic Foundation for a transesophageal echocardiography, finding in the aortic root to the posterior aspect a mass with cystic appearance, trabeculated, with dynamic flow and inside it the presence of thrombus, with an aortic valve sclerosis with mild insufficiency, without stenosis, The patient was remitted for a multidisciplinary management by cardiology, neurology and cardiovascular surgery. During the hospitalization, a chest angiography was performed, reporting multilobed dilatation of the non-coronary Valsalva sinus with a mobile thrombus inside. On the 22nd day of admission surgical correction of the aneurysm was performed through aortotomy, locating an orifice that feeds it below the commissure. The coronary and right coronary valves without communication with the right atrium with a Dacron patch. During the postoperative period she presented signs of low cardiac output and myoclonus the patient was managed with inotropes, levosimendan and levetiracetam, 48 hours after surgery she was extubated and 12 days after surgery she is discharged without complications.
\end{abstract}

Keywords: Stroke; Thrombus; Valsalva sinus aneurysm

\section{Introduction}

A transesophageal echocardiogram is an alternative way to perform an echocardiogram. Specialized probe containing an ultrasound transducer at its tip is passed into the patient's esophagus. This allows image and Doppler evaluation which can be recorded. Before inserting the probe, mild to moderate sedation is induced in the patient to ease the discomfort and to decrease the gag reflex, thus making the ultrasound probe easier to pass into the esophagus. It would help in the diagnostic processes. The advantage is usually clearer images, especially of structures that are difficult to view transthoracically. It helps to detect and quantify the disease preoperatively as well as to assess the results of surgery immediately after the procedure.

\section{Case Report}

This is a case report of a 54-years-old female with a history of systemic arterial hypertension treated with enalapril $20 \mathrm{mg}$ and amlodipine $5 \mathrm{mg}$ both once daily. She was admitted 15 days ago in a Hospital Center of Bogotá for having presented sudden loss of consciousness followed by complex focal seizure episodes. She presented with motor and sensory aphasia and left hemiparesis, atrial fibrillation was documented in electrocardiogram and an ischemic cardiovascular event was documented in a brain computer tomography. A cardioembolic event due atrial fibrillation was considered and for that reason the patient was referred to our institution for a transesophageal echocardiography, finding in the aortic root to the posterior aspect a mass with cystic appearance, trabeculated, with dynamic flow and inside it the presence of thrombus, with an aortic valve sclerosis with mild insufficiency, without stenosis, mitral valve of rheumatic aspect with mild insufficiency, severe concentric hypertrophy of the left ventricle with mild generalized hypokinesia and mild systolic dysfunction with an ejection fraction of $48 \%$, severe left atrial dilatation without thrombi, rest of valves were normal, a diffuse pericardial effusion of $80 \mathrm{ml}$ without hemodynamic repercussion was documented, it was established that the patient presented an ischemic acute Cerebrovascular event in the right frontotemporal region originated by thrombus formed in high flow trabecular mass located in aortic root (Figure 1). The patient was remitted multidisciplinary management by cardiology, neurology and cardiovascular surgery. During the hospitalization, a chest angiography was performed, reporting multilobed dilatation of the non-coronary Valsalva sinus with a mobile thrombus inside. On the 22nd day of admission surgical correction of the aneurysm was performed through aortotomy, locating an orifice that feeds it below the commissure. the coronary and right coronary valves without communication with the right atrium with a Dacron 
Citation: Somoza CF, Zuluaga JF, Rodriguez J (2018) Transesophageal Echocardiogram as a Diagnostic Tool for Cardioembolic Cerebrovascular Event Secondary to Thrombus into Valsalva Sinus Aneurysm. J Cardiovasc Dis Diagn 6: 328. doi: $10.4172 / 2329-9517.1000328$

Page 2 of 4

patch. During the postoperative period she presented signs of low cardiac output and myoclonus the patient was managed with inotropes, levosimendan and levetiracetam, 48 hours after surgery she was extubated and 12 days after surgery she is discharged without complications and without presenting new neurological additional alterations. The result of the anatomopathological study confirmed the preliminary diagnosis by images, dense fibro-connective tissue was found, with sclerosis without inflammatory compromise and the presence of hemosiderophages due to old hemorrhage (Figure 2).

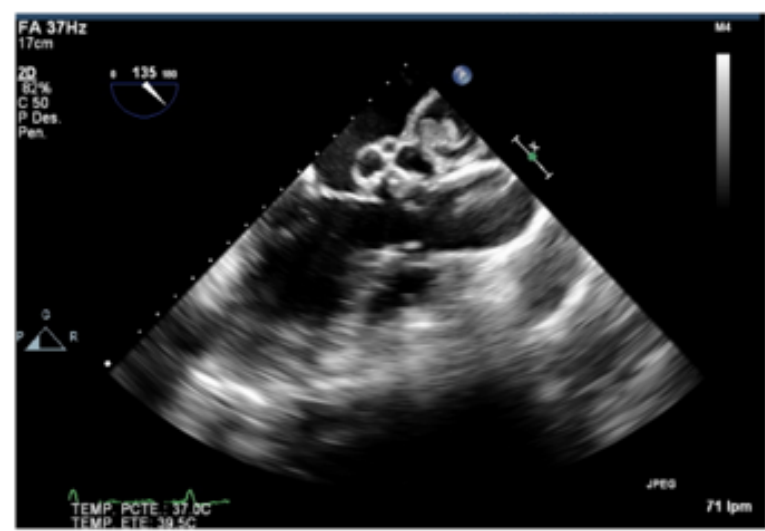

Figure 1: Mid-esophageal long axis view, a trabeculated, cystic aspect mass with flow is observed associated with right coronary Valsalva sinus (RCVS), a thrombus well organized with high embolic potential emerging from RCVS is pointed with the arrow (performed with an iE33 Philips ultrasound machine).

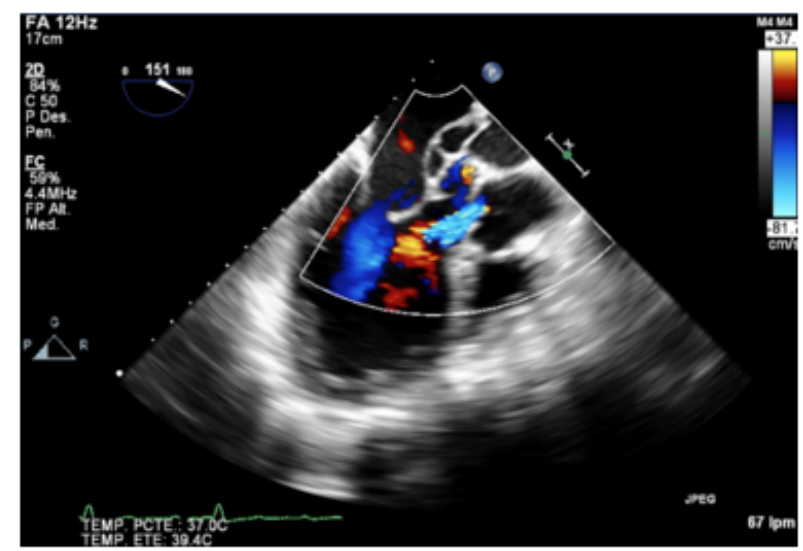

Figure 2: Mid-esophageal long axis view, a trabeculated, cystic aspect mass with flow is observed associated with right coronary Valsalva sinus (RCVS), a mild aortic valve insufficiency is associated (performed with a iE33 Philips ultrasound machine).

\section{Discussion}

Stroke is defined as an episode of neurological dysfunction (in brain, retina or spinal cord) presumed to be caused by ischemia or hemorrhage that persists for more than 24 hours or until death without has been able to perform studies necessary to classify it in any of the following groups: ischemic, intracerebral hemorrhage, subarachnoid hemorrhage, cerebral vein thrombosis, in addition to presentations that do not have acute symptoms such as silent infarcts of the central nervous system and silent cerebral hemorrhages [1]. The transient cerebral ischemic attack was defined in 2009 as brief episodes of neurological dysfunction due to focal cerebral ischemia not associated with permanent infarction [2].

According to world statistics of the World Health Organization, cardiovascular diseases (CVD) were the first cause of mortality in 2015 with 17.6 million deaths worldwide, with cerebrovascular events the second cause of mortality with 6.2 million deaths 53.3 and $46.7 \%$ respectively [3].

In the United States of America, the estimated prevalence of cardiovascular diseases is more than 85 million people ( 1 of every 3 habitants if the totally of 80 million of hypertensive patients are included), of these 15.5 million have coronary heart disease and 6.6 million patients with stroke. Among the different ethnic groups nonHispanic whites have a prevalence of $11.1 \%$ of cardiovascular disease, African Americans $10.3 \%$ and Latinos $7.8 \%$, with a prevalence of any type stroke of $2.3 \%, 4 \%$ and $2.4 \%$ respective. The prevalence in the general population of stroke in the US during the years 2009-2012 according to NHANES were 2.6\%; 2.7\% according the 2013 BRFSS (CDC). In USA 610 thousand new events/year and 185 thousand recurring events/year $7 \%$ were produced. Over the total of documented stroke $80 \%$ are ischemic, $10 \%$ intracranial hemorrhages and $3 \%$ subarachnoid hemorrhages, $6 \%$ to $28 \%$ of the population have presented silent ischemia, increasing the incidence with age and $2.3 \%$ transient ischemic attacks [4].

In Colombia the prevalence of stroke during 1984 to 2002 were between $0.1 \%$ and $1.9 \%$, the majority of them were ischemic, prevalence in 2014 among women were $0.15 \%$ for an incidence of 7.75 cases per 100 thousand women and men the prevalence was $0.17 \%$ and the incidence of 17.08 cases per 100 thousand men; hemorrhagic stroke had a prevalence among women of $0.05 \%$ with an incidence of 7.58 per 100 thousand habitants and among men a prevalence of $0.06 \%$ and an incidence of 9.08 per 100 thousand habitants [5].

The TOAST trial considered 5 etiologic subgroups of ischemic stroke:

1) Large arteries.

2) Cardio-embolism.

3) Small arteries occlusion (lacunar).

4) Stroke of another determined etiology.

5) Cryptogenic stroke due 2 or more identified cause [6].

$30 \%$ to $40 \%$ of cardioembolic stroke has a cryptogenic origin, $15 \%$ to $40 \%$ of those has cardioembolic origin [7]. The TOAST study identifies high-risk sources of cardio embolism such as the presence of mechanical valvular prostheses, mitral stenosis and concomitant atrial fibrillation, isolated atrial fibrillation, thrombi in the left atrium and atrium, sick sinus syndrome, recent minor myocardial infarction within 4 weeks, thrombi in the left ventricle, dilated cardiomyopathy, left ventricular akinesia, atrial myxoma, infectious endocarditis and medium-risk sources such as mitral valve prolapse, mitral ring calcification, mitral stenosis without atrial fibrillation, spontaneous echo contrast in the left atrium, aneurysm of the interatrial septum, permeable foramen ovale, biological valvular prosthesis, non-bacterial thrombotic endocarditis, heart failure, hypochondrial segment in left ventricle and myocardial infarction greater than 4 weeks and less than 
Citation: Somoza CF, Zuluaga JF, Rodriguez J (2018) Transesophageal Echocardiogram as a Diagnostic Tool for Cardioembolic Cerebrovascular Event Secondary to Thrombus into Valsalva Sinus Aneurysm. J Cardiovasc Dis Diagn 6: 328. doi: $10.4172 / 2329-9517.1000328$

Page 3 of 4

6 months [6] Among the possible causes of ischemic cardioembolic stroke atrial fibrillation (AF) is especially important because is fully identified as an independent risk factor that increases mortality twice in women and 1.5 times in men, increasing up to 5 times the probability of stroke. AF was by documented between $20 \%$ to $30 \%$ before or after an ischemic stroke [8,9], with a global prevalence in 2010 estimated in 20.9 million men and 12.6 million women and with an estimated incidence in 2010 of 2.7 million new cases in men and 2 million new cases in women $[10,11]$. However, in this particular case the presence of thrombi in the left atrium or in the left atrium was discarded using in the transesophageal echocardiogram but identifying a multilobulated dilation of the non-coronary Valsalva sinus with thrombus inside it an uncommon case reported in the literature.

The Valsalva sinus aneurysm (VSA) is a rare finding present among $0.14 \%$ to $0.35 \%$ of the patients undergoing to cardiac surgery $[11,12]$ with a prevalence of $0.09 \%$ in the general population [13] in autopsies series 7 cases were reported among 8138 patients [14]. VSA is defined as a dilation between the aortic root ring and the sino-tubular Groove, VSA could be acquired or congenital, among the acquired causes are infections such as syphilis [15], bacterial endocarditis, tuberculous or even fungal endocarditis or thoracic trauma [13,16,17] Congenital causes are secondary to weakness of the elastic lamina in its junction with the middle aorta and fibrous ring, is frequently associated with Marfan syndrome, Ehlers-Danlos syndrome or other connective tissue defects, as well as Takayasu arteritis or Behcet's disease [13].

Aneurysms of the sinus of Valsalva have been associated with defects of the interventricular septum (30\% of patients), aortic insufficiency $(20 \%$ to $30 \%)$, bicuspid aorta $(10 \%) \quad[11,18,19]$. Embryologically, aneurysms develop with a blind diverticulum secondary to pressure forces on the aorta, potentiated by congenital connective tissue defects $[15,16]$; congenital defects are usually small, with projections similar to fingers or diverticula that emerge from the lower portion of the sinus; acquired aneurysm are larger with an appearance of diffuse dilations of the sinus as in syphilitic aneurysms or irregular cavities communicating with the sinuses as in aneurysms that complicate valvular endocarditis or in those that produce dissection [17]. With respect to the anatomical location most are in the right coronary sinus (72\%), noncoronary sinus $22 \%$ and $6 \%$ left coronary sinus [16], most sinus aneurysms rupture drain to the right ventricle followed by right atrium [16,17]. When VSA are diagnosticated could present with rupture or not. Most of the ruptures of VSA occurs in patients between 20 and 40 years old, the initial clinical presentation is varied from asymptomatic patient to serious symptoms, there is no specific symptoms due to this pathology, the diagnosis is performed using imaging studies. The most frequent symptom is dyspnea, follow be substernal chest pain and abdominal pain. The most frequent clinical sign is a continuous murmur [16,17], VSA could simulate acute myocardial infarction [20], endocarditis on the tricuspid valve [21]. the treatment of the Valsalva aneurysms will depend on its location, risk of complications and individual risk of the patient, the surgical approach describing a low mortality with survival of $94 \%$ in 10 years, $90 \%$ at 15 years [22] with a mortality early $1.9 \%$ [23].

\section{Conclusion}

Most of the ruptures of VSA occurs in patients between 20 and 40 years old, the initial clinical presentation is varied from asymptomatic patient to serious symptoms, there is no specific symptoms due to this pathology, the diagnosis is performed using imaging studies. The most frequent symptom is dyspnea, follow be substernal chest pain and abdominal pain. The most frequent clinical sign is a continuous murmur, VSA could simulate acute myocardial infarction, endocarditis on the tricuspid valve. the treatment of the Valsalva aneurysms will depend on its location, risk of complications and individual risk of the patient, the surgical approach describing a low mortality with survival of $94 \%$ in 10 years, $90 \%$ at 15 years with a mortality early $1.9 \%$.

\section{References}

1. Sacco RL, Kasner SE, Broderick JP, Caplan LR, Connors JJ, et al. (2013) An updated definition of stroke for the 21st century: A statement for healthcare professionals from the American Heart Association/American Stroke Association. Stroke 44: 2064-2089.

2. Easton JD, Saver JL, Albers GW, Alberts MJ, Chaturvedi S, et al. (2009) Definition and evaluation of transient ischemic attack: A scientific statement for healthcare professionals from the American Heart Association/American Stroke Association Stroke Council; Council on Cardiovascular Surgery and Anesthesia; Council on Cardio. Stroke 40: 2276-2293.

3. Organization WH (2016) World health statistics 2016: Monitoring health for the SDGs Sustainable Development Goals: World Health Organization.

4. Mozaffarian D, Benjamin EJ, Go AS, Arnett DK, Blaha MJ, et al. (2016) Heart disease and stroke statistics-2016 Update: A report from the American Heart Association. Circulation 133: e38-360.

5. National Institute of Health ONdS. (2015) Fifth ONS Report: Burden of disease due to chronic noncommunicable diseases and disability in Colombia. Bogotá: National Printing of Colombia.

6. Adams HP, Bendixen BH, Kappelle LJ, Biller J, Love BB, et al. (1993) Classification of subtype of acute ischemic stroke. Definitions for use in a multicenter clinical trial. TOAST Trial of Org 10172 in Acute Stroke Treatment. Stroke 24: 35-41.

7. Saric M, Armour AC, Arnaout MS, Chaudhry FA, Grimm RA, et al. (2016) Guidelines for the use of echocardiography in the evaluation of a cardiac source of embolism. J Am Soc Echocardiogr 29: 1-29.

8. Wolf PA, Abbott RD, Kannel WB (1991) Atrial fibrillation as an independent risk factor for stroke: The Framingham study. Stroke 22: 983-988.

9. Kirchhof P, Benussi S, Kotecha D, Ahlsson A, Atar D, et al. (2016) 2016 ESC guidelines for the management of atrial fibrillation developed in collaboration with EACTS. Eur Heart J 37: 2893-2962.

10. Chugh SS, Havmoeller R, Narayanan K, Singh D, Rienstra M, et al. (2014) Worldwide epidemiology of atrial fibrillation: A global burden of disease 2010 Study. Circulation 129: 837-847.

11. Sarıkaya S, Adademir T, Elibol A, Büyükbayrak F, Onk A, et al. (2012) Surgery for ruptured sinus of Valsalva aneurysm: 25-year experience with 55 patients. Eur J Cardiothorac Surg 43: 591.

12. Moustafa S, Mookadam F, Cooper L, Adam G, Zehr K, et al. (2007) Sinus of Valsalva aneurysms: 47 years of a single center experience and systematic overview of published reports. Am J Cardiol 99: 1159-1164.

13. Feldman DN, Roman MJ (2006) Aneurysms of the sinuses of Valsalva. Cardiology 106: 73-81.

14. Smith WA (1914) Aneurysm of the sinus of valsalva: With report of two cases. J Am Med Assoc 32: 1878-1880.

15. Venning GR (1951) Aneurysms of the sinuses of Valsalva. Am Heart J 42: 57-69.

16. Batiste C, Banzal RC, Razzouk AJ (2004) Echocardiographic features of an unruptured mycotic aneurysm of the right aortic sinus of Valsalva. J Am Soc Echocardiogr 17: 474-477.

17. Greiss I, Ugolini P, Joyal M, Bouchard D, Mercier LA (2004) Ruptured aneurysm of the left sinus of valsalva discovered 41 years after a decelerational injury. J Am Soc Echocardiogr 17: 906-909.

18. Weinreich M, Yu PJ, Trost B (2015) Sinus of Valsalva Aneurysms: Review of the literature and an update on management. Clin Cardiol 38: 185-189. 
Citation: Somoza CF, Zuluaga JF, Rodriguez J (2018) Transesophageal Echocardiogram as a Diagnostic Tool for Cardioembolic Cerebrovascular Event Secondary to Thrombus into Valsalva Sinus Aneurysm. J Cardiovasc Dis Diagn 6: 328. doi: $10.4172 / 2329-9517.1000328$

Page 4 of 4

19. Bricker AO, Avutu B, Mohammed THL, Williamson EE, Syed IS, et al (2010) Valsalva sinus aneurysms: Findings at CT and MR imaging. Radiographics 30: 99-110.

20. Hoda M, Verma A, Alapati S, Alapati S, Yarrabolu TR (2017) Diagnostic paradox: Ruptured aneurysm of sinus of Valsalva simulating tricuspid valve endocarditis. Echocardiography 34: 465-467.

21. Herrador JA, Aragon V, Fernandez JC (2017) Thrombosis of a valsalva sinus aneurysm causing AMI. Rev Esp Cardiol 71: 1-44.
22. Yan F, Huo Q, Qiao J, Murat V, Ma S (2008) Surgery for sinus of valsalva aneurysm: 27-year experience with 100 patients. Asian Cardiovasc Thorac Ann 16: 361-365.

23. Vural KM, Şener E, Taşdemir O, Bayazıt K (2001) Approach to sinus of valsalva aneurysms: A review of 53 cases. Eur J Cardiothorac Surg 20: 7176. 